Itinéraires Itinéraires

Littérature, textes, cultures

\title{
Un sens en moins, du sens en plus : une lecture de Rapport sur moi de G. Bouillier
}

Anne Coudreuse

\section{OpenEdition}

\section{Journals}

Édition électronique

URL : http://journals.openedition.org/itineraires/1214

DOI : $10.4000 /$ itineraires. 1214

ISSN : 2427-920X

Éditeur

Pléiade

\section{Édition imprimée}

Date de publication : 1 décembre 2009

Pagination : 163-174

ISBN : 978-2-296-10791-5

ISSN : 2100-1340

\section{Référence électronique}

Anne Coudreuse, «Un sens en moins, du sens en plus : une lecture de Rapport sur moi de G. Bouillier », Itinéraires [En ligne], 2009-4 | 2009, mis en ligne le 02 septembre 2014, consulté le 19 avril 2019. URL: http://journals.openedition.org/itineraires/1214; DOI : 10.4000/itineraires.1214

Ce document a été généré automatiquement le 19 avril 2019

\section{(c)}

Itinéraires est mis à disposition selon les termes de la licence Creative Commons Attribution - Pas d'Utilisation Commerciale - Pas de Modification 4.0 International. 


\title{
Un sens en moins, du sens en plus : une lecture de Rapport sur moi de G. Bouillier
}

\author{
Anne Coudreuse
}

1 Rapport sur moi est le premier livre publié par Grégoire Bouillier, d'abord aux éditions Allia en 2002, repris en «J'ai lu » en 2004, après avoir obtenu le prix de Flore. C'est un récit déconcertant, mis ironiquement sous la protection du Prince de Ligne, un brillant aristocrate des Lumières, cité en épigraphe : « Un des ouvrages de Diderot tomba entre les mains de Frédéric II. L'empereur y trouva d'emblée ces paroles : Aux jeunes gens... Sur ce, il ferma le livre, comprenant bien qu'il ne s'adressait pas à lui. » La séduction opère dès le début comme un refus de la séduction, un contrat de lecture fondé sur le paradoxe d'un livre qui s'ouvre par un livre qui se ferme. L'épigraphe entre aussi en tension avec la première phrase du récit : «J'ai vécu une enfance heureuse ${ }^{1}$. » Cet incipit n'est guère celui qu'on attendrait dans un livre dont le titre, dans sa froideur clinique ou policière, suggère un certain nombre de comptes à régler, un dossier instruit à charge autour de pièces d'un «moi » qui n'aurait pas su convaincre, avec lequel l'auteur entretiendrait un « rapport » distant et ironique, dont tout reste à dire. De l'anosmie qui frappe le narrateur dans son enfance au délire qui lui fait lire sa vie dans l'odyssée à la suite d'une rupture amoureuse, la question des sens et du sens se pose de façon particulièrement troublante dans ce récit à la fois sec et émouvant, l'émotion qu'il suscite étant à la mesure de la précision d'une plume tenue comme un scalpel, qui incise les expériences et les mots avec une ironie conçue comme lucidité et refus de s'appesantir sur une douleur, qui dans tout le récit, va défaire et déconstruire l'incipit évident qui peut aussi fonctionner comme une antiphrase. C'est alors le sens même de la démarche particulière qui consiste à écrire sur soi qui se met à trembler dans cette réflexion sur l'écriture et ses mirages.

2 Ce qui frappe dans le livre, c'est sa composition, qui ne respecte pas la chronologie et propose une sorte de boucle : il s'ouvre et se referme sur une tentative de suicide de la mère. La dernière phrase ne peut être qu'un écho à l'incipit : «Je lui dis que c'est encore 
heureux» (p. 127). Entre ces deux évocations ironiques du bonheur, et la répétition du même adjectif, le narrateur procède à des concaténations de différents épisodes de sa vie qu'il éclaire les uns par les autres. On peut recomposer le centre du motif dans une scène de disparition alors qu'il a neuf ans :

Depuis ce dimanche de 1969, les aléas du moi me sont toujours apparus piteux. [...] Il me faudrait un jour payer ma dette pour avoir pu approcher l'intime prestidigitation du monde, l'absolu dégingandé de la vie.

J'ai payé. (p. 74)

Cette disparition est celle de la famille Fenwick: "mon meilleur ami, mon amour d'enfance et leur mère la déesse » (p. 73), qu'il a vue nue, «Ingres et Delacroix réconciliés rien que pour moi dans une salle de bains » (p. 66). L'impression d'éclats de sens, sinon d'éclatement, est bien rendue par une particularité typographique du livre, qui progresse en une série de paragraphes espacés les uns des autres par un blanc, et regroupés en chapitres non numérotés. De là, le sens est à construire autant dans les ellipses que dans les micro-scènes dessinées cruellement, la plus représentative étant celle de la conception du narrateur :

Là-bas ils s'aimèrent. Et plutôt trois fois qu'une, puisqu'un interne de l'hôpital de Tizi-Ouzou tomba sous le charme de ma mère, qui n'en manquait pas; bientôt il devait se joindre à leurs ébats; c'est lors d'une de leurs parties à trois que je fus conçu. (p. 8)

Il suffit d'une variation ironique sur une expression figée de la langue française, "plutôt deux fois qu'une» devenant "plutôt trois fois qu'une", pour que le sens se mette à trembler dès l'origine, puisque ce trio aurait donné naissance à un «mutant» (p. 9), en tout cas d'après ce qu'a lu la mère dans un magazine... Cette hésitation sur le père, et en particulier sur son nom, reprend, mais autrement, un même brouillage à la génération des grands-parents :

Ma mère avait de qui tenir : elle allait sur ses douze ans lorsque son frère de deux ans son aîné se leva de table et lança au père qui le réprimandait pour une peccadille: «Tu n'es pas notre vrai père!» De fait, il s'agissait de leur oncle, qui avait secrètement pris dans le lit de sa belle-sœur la place que son frère y occupait avant de disparaitre dans les premiers temps de la Seconde Guerre Mondiale. [...] D'un frère l'autre, ma mamie vivait toujours avec un Pérard et elle n'eut pas à changer de nom pour continuer d'apparaître aux yeux du monde merveilleusement mariée. (p. 10)

Le délire que le narrateur décrit vers la fin de son récit pourrait alors s'appréhender en termes psychanalytiques, si on le met en rapport avec ce désordre initial, ce que Grégoire Bouillier se garde bien de faire. On peut ainsi renvoyer à la conclusion d'un livre de psychanalyse qui prend en compte les conceptions lacaniennes :

Quand le mot dit tout, quand il croit saisir pleinement la chose, ce qui s'avère défaillant est un accès à la perte inhérente à la significantisation. La fonction de représentation de cette perte dans le champ du signifiant est dévolue au phallus. La thèse freudienne du retrait de l'investissement psychique des objets est congmente avec la carence de la signification phallique désignée comme conséquence majeure de la forclusion du Nom-du-Père ${ }^{2}$.

4 Loin de ces élaborations théoriques, le narrateur préfère s'en tenir à un éclairage sémantique et étymologique quand il conclut, sur une pirouette, ce chapitre originel et original :

En vieux français, Pérard signifie «mauvais père ». Bouillier, pour sa part, désigne un " petit bois de bouleaux ». Je sais donc de quel bois je suis fait, ce qui n'est pas donné à tout le monde. (p. 10) 
De son père, réel ou putatif, il ne saura rien que le regard de sa mère sur lui : « De fait, ma mère refusa de rester en Grande Kabylie, bien que son amant l'en suppliât, et le trio se sépara, pour ne plus exister que dans les yeux de ma mère lorsqu'elle me regarde " (p.13). C'est aussi évidemment du côté de la mère qu'il faut chercher les origines de ce délire, comme le montrent la scène inaugurale, juste après l'incipit, et la suite du récit. Ce qui provoque la psychose, c'est le caractère invivable du double bind :

Depuis un article de 1956, signé par Gregory Bateson et un groupe de collègues, on commence en fait à imputer la schizophrénie (et le délire) à l'effet de "doubles liens ", c'est-à-dire à des messages qui s'annulent réciproquement ou à des ordres inexécutables. [...] En effet, quand une mère prétend aimer son enfant, mais ne veut pas le laisser grandir comme une personne indépendante, en réalité « elle désire l'enfant en soi, elle ne l'aime pas pour ce qu'il est; il doit satisfaire ses besoins profonds d'intégrité, de pureté et de tendresse (d'être aimée); il ne lui est pas permis de se soustraire à la fonction qui lui est imposée, et donc, avant toute chose, il ne doit pas grandir trop ni ne peut devenir autonome ». À l'enfant sont adressés des messages contradictoires du type: «Je (ne) t'aime (pas) - Je (ne) m'aime (pas)». Le caractère paradoxal de ces messages peut se résumer dans le commandement: "Sois ce que tu n'es pas!», l'exact contraire du précepte classique : «Deviens ce que tu es !», formulé par Pindare et par Aristote, puis repris par Nietzsche ${ }^{3}$.

6 L'inconfort troublant du double lien est restitué par les moyens de la syntaxe, dans l'absence de suture entre les différents paragraphes qui donnent l'impression d'éclats indépendants les uns des autres, comme si l'auteur disposait d'une grammaire sans aucun outil logique. On peut en donner l'exemple suivant, pris presque au hasard:

Lorsque je sortis du ventre de ma mère, il parait que je riais. Les sages-femmes se disputaient presque pour s'occuper de moi : jamais elles n'avaient vu bébé si joyeux de faire son entrée dans la vie.

Trois jours plus tard, je pesais moins d'un kilo et mon état épouvantait. [...] On crut que je ne passerais pas une journée de plus lorsque je condescendis à absorber le lait d'une chèvre que l'on avait trouvée presque par hasard dans les environs de la maternité. C'est à cet animal à la réputation de sale caractère que je dois d'avoir survécu. (p. 11-12)

7 C'est au lecteur de rétablir les liens manquants, dans ce puzzle où même le sexe du narrateur semble être simplement dû à un caprice arbitraire, à une velléité maternelle qui le concernerait assez peu :

Comme pour son premier fils, ma mère n'a jamais douté qu'elle attendait un garçon. "Je suis incapable d'avoir une fille», dit-elle avec fierté. Ce qui ne l'empêchait pas de me friser les cheveux avec son Babyliss quand l'envie lui prenait. (p. 12)

Il devient le héros improbable d'une légende familiale qu'on lui répète sans qu'il la comprenne :

«Vous voulez qu'on lui coupe?»[...] Personne ne songe à m'expliquer qu'il s'agissait de me circoncire. Ce qui ne change rien puisque ma mère riait précisément du quiproquo. Mais moi, j'ai frémi des années durant que l'on puisse décider du sexe des enfants après leur naissance et que je devais au seul bon vouloir de ma mère d'être un garçon et non une fille. Si elle avait dit oui ? (p. 22)

8 Puisque le monde ne semble pas avoir de sens, il va chercher à le décrypter dans ses signifiants. C'est ainsi qu'il voit dans sa date de naissance, qu'il écrit «de manière algébrique » 2206 60, « un mystérieux palindrome arithmétique me distinguant cette fois favorablement du lot » (p. 14). Aucun fétichisme narcissique, ne serait-ce que dans l'ordre des informations puisque la date de naissance n'est pas donnée à l'entrée du récit, comme 
dans les modèles canoniques de l'autobiographie, en particulier dans les Confessions de Rousseau. De même, s'il évoque souvent de grandes douleurs et des figures parentales très défaillantes, ce n'est pas le ton du règlement de compte qui l'emporte, l'auteur n'en faisant qu'une boutade qui l'épuise et le dénonce comme artifice infantile, lorsqu'il évoque une gifle de sa nourrice qui l'a fait tomber de sa chaise de bébé, ce qui lui a fendu le front en deux :

Il n'est pas rare que des gens qui me connaissent pourtant depuis longtemps s'étonnent de cette blessure que j'ai au front. Ils ne l'ont jamais remarquée auparavant et la croient récente. J'explique alors que c'est ma mère qui m'a mordu. (p. 18)

Sur l'inconséquence de ses géniteurs et les circonstances de sa conception, il ne porte aucun jugement moral, comme s'il ne gardait aucune amertume de ce trouble de l'identité, et était plutôt reconnaissant à ses parents de l'inscrire dans une filiation littéraire :

De même, lorsque les temps furent à la prétendue "libération sexuelle ", j'en étais déjà le fruit, mes parents n'ayant eu besoin d'aucun mot d'ordre pour jouir sans entraves. Boccace et Aristophane ont toujours été pour moi proches de la vérité, ainsi que Sade et Georges Bataille, mais ce dernier surtout parce qu'il porte les mêmes initiales que moi. (p. 13)

C'est bien celui à qui, à la suite d'une rupture amoureuse, tout « faisait signe » (p. 84), qui relit sa vie autant qu'il la relie à des figures tutélaires qu'il choisit en dehors de sa famille. Cette maladie du sens, cherchant dans le signifiant même l'origine et l'explication d'un destin, en refusant toute psychologie héritée d'un certain roman, semble également avoir frappé le frère du narrateur qui fait le lien entre « son goût pour les hommes » et le nom de leur nourrice, « Madame Guillaumot » : «son premier amant s'appelait Guy » (p. 18). C'est une figure presque absente du récit, où il occupe pourtant des places stratégiques, par le caractère glauque des évocations qui en sont faites. Le mot « inceste » n'est bien évidemment pas écrit, mais parfaitement décrit, dans son caractère absolument inouï.

Là encore, pas un seul jugement moral, mais une clausule de chapitre qui en dit plus long que tous les romans de Christine Angot: « De ce jour, je n'ai plus souvenir que nous nous soyons adressé la parole comme deux frères"(p.51), qui fait écho à la formule, syntaxiquement très proche, qui clôt le chapitre précédent : « Dès cet instant, je ne lui dis d'ailleurs plus rien ; elle a cessé d'être ma mère, même si je reste son fils » (p. 43). Le frère ne refait une apparition dans le récit que pour mourir du SIDA, avec un refus du pathos qui fait que le lecteur ne peut même pas s'y accrocher pour évacuer l'angoisse qui provient de la sécheresse de cette écriture sans un pli : «Mon frère n'eut pas la force de passer une trente-troisième fois par la case départ : il mourut dans la nuit » (p. 55).

Cette attention au signifiant permet de donner une dimension fondatrice aux "staphylocoques dorés » que le narrateur contracte dans sa petite enfance, « en buvant de l'eau croupie ", sans doute, selon ses parents « en léchant la vitre du train qu'il fallait prendre, chaque dimanche soir, pour revenir de chez mes grands-parents» (p.22). Immédiatement, il établit un rapport, comme le suggère le titre, avec la jeune fille qu'il rencontra dans un train, vingt-cinq ans plus tard, en revenant de Berlin : « Elle s'appelait Laurence, faute peut-être que "l'eau croupie" soit un prénom » (p. 23). Et toute l'histoire entre eux semble se résumer à une question de grammaire et de vocabulaire, ce qui ne lui enlève rien de sa violence et de sa douleur, au contraire :

Ce qu'elle faisait de la langue m'étranglait plus que tout. Elle avait des mots plein la bouche, et des plus ronflants, des plus universels, qu'elle jetait en l'air sans souci de 
savoir où ils retombaient. [...]

Malgré ses petits seins et sa délicate fleur de peau, je ne pouvais souffrir sa syntaxe. Quand elle parlait, elle était des millions et c'était trop de monde pour moi. (p. 25-26)

Établissant la liste des prénoms des femmes qu'il a aimées, le narrateur note, dans ce qui n'est pas qu'une boutade : « Pour moi, l'amour est aussi une affaire de voyelles » (p. 28).

Ces paragraphes qui entretiennent entre eux un si curieux "rapport» (comme est étrange le « rapport » du narrateur à ses parents et au monde) sont cependant organisés selon un art, aussi savant que secret ou discret, de la composition dramatique. C'est ainsi qu'apparaît dans le premier quart du récit « cette plante moly dont il est dit dans l'Odyssée qu'elle protégea Ulysse de la puissance sexuelle de Circé, la magicienne qui transformait en pourceaux les marins accostant sur son île» (p. 27). C'est l'effet de la "joie » d' « une certaine Anastasie-Louise » qui sauve le narrateur de la détresse où l'a laissé Laurence / "l'eau croupie ». Or toute la fin du récit est consacrée à la lecture de l'Odyssée qui sauve le narrateur du délire par un délire plus convaincant encore :

Mais ils [mes parents] ne pouvaient toujours rien pour moi. Ni la télévision, que je ne songeais pas à regarder dans l'espoir d'y trouver, comme à neuf ans, une explication à ce qui m'était arrivé. J'avais trouvé mieux, qui était pourtant le même miracle dont j'avais besoin: l'odyssée d'Homère, que je lus en une seule nuit transfigurée.

Jamais auparavant je n'avais connu semblable expérience avec un livre, et par la suite non plus. C'était comme si j'offrais mon visage au soleil. Chaque vers semblait écrit à mon intention et s'infusait en moi, s'écoulant par mes yeux et mes oreilles. J'étais la lecture même.

Ou plutôt, c'était l'Odyssée qui me déchiffrait. Car tout s'éclairait soudain à sa lumière. D'inouïes coïncidences surgissaient entre ce que je lisais et ce que je vivais, les frontières étaient abolies et je pouvais voir entre les lignes par où moi-même étais passé. En filigrane des aventures d'Ulysse se révélaient les miennes, non pas identiques, mais reprises. Charybde et Scylla, les bœufs du Soleil, le cyclope... j'avais, à ma manière, vécu tout cela. Je pouvais citer les lieux et les dates. Renouer les fils. [...] Alors l'Odyssée était l'oracle qui m'enseignait mon avenir... Il me fallait parfois poser le livre pour reprendre ma respiration. (p. 87-88)

13 Ce qui est frappant et émouvant, c'est le refus du narrateur de médicaliser a posteriori son expérience, en en donnant par le récit rétrospectif une explication rationnelle, qui établirait des rapports entre cette lecture et sa vie. Il la maintient dans la présence éblouissante et singulière de sa découverte salvatrice, notant un peu plus loin: «Pourquoi me serais-je laissé convaincre que je devais me faire soigner si c'était pour souffrir un désespoir dont on ne peut faire aucun usage qui ne soit avilissant pour soi ou les autres?» (p. 89). Quiconque connaît un peu les théories psychanalytiques reconnaîtra ici la fonction curative du délire : «la thèse freudienne selon laquelle il constitue une tentative de guérison n'est pas ignorée ${ }^{4}$ ", signale Jean-Claude Maleval dès les premières lignes de sa réflexion, avant de développer cette idée :

C'est en 1911, à l'occasion de son commentaire du livre du président Schreber, que Freud conçoit ce dont nul autre avant lui n'avait clairement eu l'intuition, à savoir que « ce que nous prenons pour une production morbide, la formation du délire, est en réalité une tentative de guérison, une reconstruction ${ }^{5}$ ».

On peut également citer cette analyse de Freud, dans la même étude : «Le paranoïaque rebâtit l'univers, non pas à la vérité plus splendide, mais du moins tel qu'il puisse de nouveau y vivre. Il le rebâtit au moyen de son travail délirant. » Remo Bodei, quant à lui, y voit une « perle difforme constituée autour d'un grumeau de souffrance ${ }^{6} »$. Son livre, dont 
le premier chapitre s'intitule "l'héritage du passé ", a le mérite de faire le lien entre l'expérience passée et un événement présent qui vient la réactiver, douleur à laquelle le délire procure un apaisement ou un dérivatif. Au sens étymologique, « délirer » vient du latin delirare qui signifie "sortir du sillon", " extravaguer », alors que lirare signifiait «tracer des sillons». Il s'agit dans l'expérience que le narrateur nous décrit dans sa singularité, moins de sortir du sillon, que de revenir en tracer un plus ancien, qui est resté douloureusement vivant dans sa conscience :

Un chef-d'œuvre de disparition! Il fallut presque m'interner. [...] Quel flair était donc le mien, moi l'anosmique, pour avoir élu parmi la multitude l'être capable de reproduire la disparition de Mme Fenwick qui, quinze ans auparavant, paraissait pourtant insurmontable? L'histoire se répète de manière caricaturale, ricanais-je tout haut dans les rues. (p. 83)

Il faut mettre cette reviviscence de la douleur en rapport avec le trauma initial qu'il réactive, en privant le monde de sens, et en obligeant le narrateur à en construire un de substitution :

Et je reste comme anesthésié, vide de vie, sans plus rien ressentir, ni sentiment, ni sensation, seulement un besoin gourd de néant, comme un brouillard m'envahissant depuis derrière les yeux, torpeur sans nom et sans température dans l'épaisseur de laquelle je me dissous, ne laissant sur place qu'une statue de moi changé en pierre qui doit encore trôner à l'insu de tous dans l'entrée. (p. 69)

Tout n'était-il pas d'une passionnante absurdité ? [...] je ne serais désormais sensible qu'aux apparitions et aux disparitions. (p. 73)

Le délire va alors être une manière de donner un sens à «l'absurdité » de la disparition nouvelle, selon un processus pour lequel les réflexions de Remo Bodei fournissent des éclaircissements :

Les psychoses surviennent quand le dé-plaisir procuré par les contenus refoulés provoque des tensions psychiques si insupportables qu'elles ne peuvent plus se manifester sous la forme de symptômes locaux de compromis, c'est-à-dire quand leur traduction dans le langage du présent échoue sur toute la ligne. Le délire est alors le résultat d'une fracture difficilement colmatable entre divers stades de l'existence, d'un tremblement de terre qui bouleverse les strates de la personnalité péniblement superposées. Un traumatisme, un stress ou un life event [...] rouvrent des plaies qui n'étaient pas entièrement cicatrisées, réactivent des désirs insatisfaits, réveillent des peurs anciennes, des sentiments de culpabilité ou des incompréhensions, mettant à découvert et élargissant les fêlures latentes et aggravant le vieux déficit de délimitation logico-affective du monde intérieur et du monde extérieur ${ }^{7}$.

On préférera sans doute les mots plus simples du narrateur, qui incarnent ce dispositif complexe :

De fait, lorsque je fus tiré d'affaire, je réalisai que, trois mois durant, j'avais eu neuf ans dans la peau d'un homme de trente ans afin de lever l'interdit où cet âge m'avait laissé. Et si une manifestation m'avait de nouveau barré le passage, il s'agissait cette fois de celle de la folie, ultime protestation de la vie lorsque la mort lui est arbitrairement imposée. Tout se transposait, s'approfondissait en variations. Les mêmes scènes se reproduisaient, mon histoire se déroulait de manière conforme, mais sous un autre angle, cosinus de l'ancien. (p. 85)

Le lecteur de Rapport sur moi est sensible au souci du narrateur de ne pas psychiatriser rétrospectivement la façon dont pour lui le sens et le monde ont tremblé. Le mot «délire» qu'il emploie n'est pas l'apanage des psychiatres et il est entré assez communément dans la langue. En revanche, il n'emploie pas les mots "psychose", «paranoïa» ou «schizophrénie». Ce refus d'un diagnostic, cette volonté de faire 
ressentir à son lecteur l'apparente logique de l'enchaînement des émotions et des sensations, restituent cette expérience singulière dans son inquiétante étrangeté et dans sa possible universalité. De même, il n'est jamais question de guérison, mais plutôt d'une intégration de cette découverte fondatrice dans la conscience et dans la vie du narrateur, et juste dans sa capacité nouvelle à la mettre en mots :

On peut en penser ce que l'on veut, mais me croire un inédit d'Ulysse valait mieux que de me prendre pour un homme moderne. Fiction pour fiction, la mienne me rendait ma liberté de mouvements. [...]

Même aux pires moments, ma vie ne m'a ainsi jamais déçu. J'avais trouvé ma formule. (p. 90)

Sans qu'il établisse ce lien explicitement, le lecteur le voit se dessiner entre l'expérience de la folie et le livre dense qu'il tient entre ses mains : «Je passais mon temps à lire et à écrire sur des carnets. De peinture il n'était plus question dans ma situation, sinon avec des mots » (p. 91). Tous les psychiatres ont décelé et souligné la nature verbale du délire et les rapports qu'il entretient souvent avec la graphomanie ou, plus dignement, avec la création littéraire :

L'importance majeure des troubles verbaux dans la psychose, cette tendance des mots à copuler entre eux, constitue un trait clinique essentiel, souligné aussi bien par Freud que par Clérambault, Lacan et plusieurs autres psychiatres avant eux ${ }^{8}$.

Le narrateur lui-même met bien en évidence cette primauté de l'écriture dans la première phase de son délire :

Lorsque les voix me laissaient tranquille, c'était alors une autre frénésie qui me saisissait : celle d'écrire tout ce qui m'arrivait dans les marges du moindre journal que je ramassais [...]. (p. 84)

Je me rappelle une phrase qu'inlassablement je griffonnais sur tout ce qui me tombait sous la main, comme un talisman que j'accrochais partout: « Le chemin s'est perdu en cours de route, c'est donc qu'il y a une route. " J'ignorais laquelle, mais cette certitude me tenait debout. (p. 85)

Cette hallucination du signifiant le poursuit bien après la fin du délire, quand il se rend en métro au chevet de sa mère qui a fait une nouvelle tentative de suicide : « Sur le quai, une publicité affiche en gros caractères "j'ai bien fait de vomir." Il faut lire "j'ai bien fait de venir." »(p.101). L'hallucination dit mieux la vérité que la "réalité» des mots effectivement affichés.

Lorsqu'il raconte les phénomènes troublants de la forme initiale de son délire, le narrateur ne cède à aucun pathos, et c'est l'humour qui domine ce compte rendu :

J'entendais tout le temps des voix, qui m'ordonnaient de tourner à gauche, ou à droite, de marcher tout droit, de hennir car un cheval avait poussé une nuit en moi (c'est immense le corps d'un cheval). (p. 83)

La distance prise grâce à l'emploi de l'imparfait est annulée par le présent de vérité générale qui apparaît dans la parenthèse, comme une règle de logique universelle. L'effet d'une telle construction d'écriture est de désamorcer l'angoisse du lecteur, en faisant de la folie une expérience à la portée de tous, et en même temps irréductiblement singulière. C'est également avec humour qu'il décrit la mégalomanie souvent associée au délire, et qu'il présente comme allant de soi, avec une sorte de gaieté qui constitue paradoxalement un des principes fondateurs de l'émotion qui émane de ce récit :

Survenant à l'improviste, les voix disparaissaient de même, me laissant pantois et, en même temps, empli d'un sentiment de puissance que rien ne pouvait altérer, ni le froid ni la faim. J'étais le maître anonyme du temps. L'élu de l'infra-monde. L'incarnation du primesaut universel. (p. 84) 
Il aura sans doute fallu à l'auteur faire l'expérience d'un sens en moins pour parvenir à donner du sens à tout ce qui en manque, ce qui pourrait être une définition de l'expérience littéraire. Privé d'odorat, à la suite de son infection par les staphylocoques dorés, il doit apprendre à compenser cette défaillance par une dépense supplémentaire d'intelligence et de langage :

J'y perdis cependant l'odorat, ce dont personne ne se rendit compte. Moi-même le dissimulai longtemps, sous couvert de stratégies que je développais.

J'affirmais par exemple avec enthousiasme que la salade sentait bon le citron après avoir surpris un pépin dans la vinaigrette. Si jamais je suis intelligent, c'est en trompant mon monde que je le suis devenu : que ne devais-je étudier les apparences pour leur donner un sens que j'avais perdu. C'est ainsi que je sus très tôt que le vraisemblable ne se confond pas avec la vérité, ni le réel avec sa représentation, ce qui m'éloigna rapidement de mon époque. Je devins d'ailleurs très tôt solitaire puisque non seulement il me fallait garder secrète mon anosmie, mais cela au milieu de gens qui ne me faisaient guère envie si je pouvais les abuser si facilement. (p. 20)

Ce qui pourrait n'être qu'un défaut pittoresque de la sensation devient le principe d'une écriture et d'une réflexion sur la poétique et l'esthétique. C'est sans doute dans ce passage, où l'on passe de l'anecdote à la portée théorique que lui donne le narrateur, que se trouve le mieux définie la poétique de ce récit original, qui fait trembler les catégories pourtant nombreuses et fines de l'écriture de soi. Elle emprunte des traits propres au roman, à l'autobiographie et à l'autofiction tellement à la mode, sans s'y réduire et s'y définir strictement. Tout se passe comme si, pour dire une expérience unique d'une odyssée en miniature et de quarante années d'une vie douloureuse commencée sous des auspices pour le moins troublants, il fallait du même geste d'écriture inventer un genre nouveau, un hybride de la poétique, un «mutant » théorique, qui dans sa singularité ne pourra pas avoir d'épigones et ne pourra pas faire école. Bref c'est une façon bien paradoxale, le talent étant alors une question de survie, de «former une entreprise qui n'eût jamais d'exemple ", plus de deux siècles après Rousseau et ses géniaux descendants. La question de la filiation est alors, on le voit bien, aussi une question d'histoire littéraire et d'écriture.

21 L'expérience littéraire est marquée au coin du mensonge, comme le montre l'anecdote suivante, qui constitue peut-être l'art poétique paradoxal de ce récit :

À l'école primaire, j'ai obtenu ma meilleure note de rédaction en racontant le souk de Marrakech, ses couleurs chatoyantes et ses odeurs enivrantes. La maîtresse lut ma copie devant tout le monde et la fit même circuler dans d'autres classes. Ce fut mon premier succès dans le monde. Il me fit bien réfléchir sur la littérature et sur l'imposture : je n'étais jamais allé à Marrakech et je n'avais pas d'odorat. (p. 20)

À partir des débris dans lesquels sa vie semblait perdre tout sens, ou en prendre trop, de manière envahissante, ce qui revient peut-être au même, Grégoire Bouillier a donc su composer un récit déroutant, qui ne suit ni le sens univoque et rectiligne de la chronologie, ni celui qu'un psychanalyste pourrait reconstruire a posteriori, dans un langage spécifique qui redonnerait sa place à une certaine logique, comme le prouvent les titres des ouvrages que nous avons consultés et utilisés. Rapport sur moi est un livre qui contient sa propre poétique, parfois dans une anecdote apparemment insignifiante qui constitue peut-être la meilleure clé pour en comprendre le sens, à la fois comme démarche et comme signification. Maniant une matière douloureuse avec humour et détachement, Grégoire incite le lecteur à interroger à son tour son propre « rapport » au sens de sa vie. Dans son deuxième livre, où la littérature joue également un très grand 
rôle, Grégoire Bouillier a cette formule infiniment séduisante et paradoxale qui dit sans doute la situation et le sens mobiles du continent des écritures de soi aujourd'hui : "Je n'invente rien car j'ai beaucoup trop d'imagination pour cela ${ }^{9}$.»

\section{NOTES}

1. Grégoire Bouillier, Rapport sur moi, Paris, Allia, 2002. Nous citons le texte dans l'édition "J'ai lu », 2004, p. 7. Les citations du texte seront suivies du numéro de page entre parenthèses.

2. Jean-Claude Maleval, Logique du délire, Paris, Masson, coll. « Ouvertures psy », 2000, p. 205. Voir aussi p. 89 : «C'est à l'occasion d'une relecture attentive des Mémoires d'un névropathe de Schreber (1903) et du commentaire qu'en fit Freud (1911) que Lacan dégagea dans son Séminaire III (1955-1956) et dans Question préliminaire à tout traitement possible de la psychose (1958-1959) le concept de forclusion du Nom-du-Père. [...] La fortune rapide connue par le concept de forclusion $\mathrm{du}$ Nom-du-Père, à partir des années 60 , réside pour une bonne part dans la constatation incontournable selon laquelle l'imaginaire paternel - différemment incarné - tient une place prépondérante dans la symptomatologie de la psychose. »

3. Remo Bodei, Logiques du délire. Raison, affects, folie, Paris, Aubier, coll. «Philosophie», 2002, p. 101-102.

4. Jean-Claude Maleval, op. cit., p. 1.

5. Ibid., p.33. La citation de Freud est extraite des "remarques psychanalytiques sur l'autobiographie d'un cas de paranoïa » (1911), dans Cinq psychanalyses, Paris, PUF, 1967, p. 315.

6. Remo Bodei, op. cit., p. 50.

7. Ibid., p. 44. Voir Freud : «Dans les délires, la folie est employée comme une pièce qu'on colle là où initialement s'était produite une faille dans la relation du moi au monde extérieur ", Névrose, psychose et perversion, Paris, PUF, 1973, p. 285.

8. Jean-Claude Maleval, op. cit., p. 38. Voir Lacan : «Les productions discursives qui caractérisent le registre des paranoïas, s'épanouissent la plupart du temps en productions littéraires, au sens où littéraires veut dire simplement feuilles de papier couvertes avec de l'écriture ", "Les psychoses », Le Séminaire III, Paris, Seuil, 1981, p. 89.

9. Grégoire Bouillier, L'Invité mystère, Paris, Allia, 2004, p. 27. Sur cette question, voir Françoise Simonet-Tenant, Le Propre de l'écriture de soi, Paris, Téraèdre, 2007.

\section{RÉSUMÉS}

Il s'agit de regarder comment Grégoire Bouillier perturbe l'écriture autobiographique par le biais d'un conte déformé qui refuse d'avoir recours à la psychanalyse, alors même qu'il raconte la folie et le désespoir tout en préservant un humour salutaire. L'intime est raconté par fragments, par évocation et par suggestions sans devenir le sujet d'analyse psychologique que l'on trouve dans un roman. Le résultat est une histoire sans pathos qui décrit ce qu'il y a de pire dans une 
existence. C'est au lecteur de reconstruire le sens en utilisant finalement certains outils psychanalytiques que l'auteur ne fournit ni n'ignore.

It is a question of contemplating how Grégoire Bouillier disturbs autobiographical writing through a distorted tale that refuses to resort to psychoanalysis, even as it recounts madness and despair while preserving salutary humour. The intimate is told through fragments, evocation and suggestions without becoming the subject of the kind of psychological analysis one picks up from a novel. The resuit is a story without pathos to describe the worst of an existence. It is up to the reader to reconstruct meaning by ultimately using certain psychoanalytical tools that the author neither provides, nor ignores.

\section{INDEX}

Mots-clés : anosmie, délire, psychose, autobiographie, écriture de soi

Keywords : anosmia, delirium, psychosis, autobiography, writing the self

\section{AUTEUR}

\section{ANNE COUDREUSE}

Université Paris 13, CENEL - IUF 